
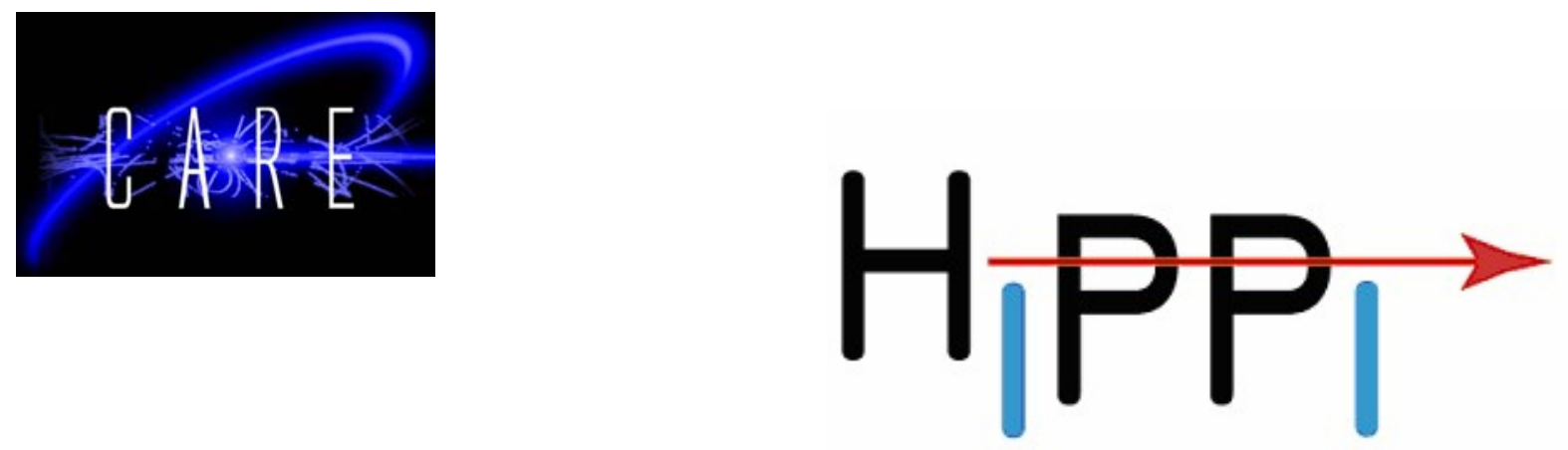

\title{
A SPACE CHARGE COMPENSATION STUDY OF LOW ENERGY HYDROGEN ION BEAMS
}

A. BenIsmaïl, R. Duperrier, D. Uriot, CEN Saclay, DSM/DAPNIA, 91191 Gif sur Yvette, N. Pichoff, CEN Bruyères-le-Châtel, DIF /DPTA, BP12, 91680 Bruyères-le-Châtel, France.

\begin{abstract}
High-power accelerators are being studied for several projects including accelerator driven neutron or neutrino sources. The low energy part of these facilities has to be carefully optimized to match the beam requirements of the higher energy parts. The complexity of high intensity beam dynamics in the low energy line is essentially due to the non-linear space charge effects. The PIC code CARTAGO [1] has been developed in order to simulate the beam transport at low energy including the temporal evolution of the space charge compensation. This paper relates new features of the code. The effects of the longitudinal space charge, the image charge and external magnetic field were included. The results of beam transports using solenoid are discussed. Space charge compensation degrees are given.
\end{abstract}

Contribution to the PAC05, Knoxville, USA

Work supported by the European Community-Research Infrastructure Activity under the FP6 "Structuring the European Research Area" programme (CARE, contract number RII3-CT2003-506395). 


\title{
A SPACE CHARGE COMPENSATION STUDY OF LOW ENERGY HYDROGEN ION BEAMS *
}

\author{
A. BenIsmail ${ }^{\#}$, R. Duperrier, D. Uriot, CEN Saclay, DSM/DAPNIA, 91191 Gif sur Yvette, \\ N. Pichoff, CEN Bruyères-le-Châtel, DIF /DPTA, BP12, 91680 Bruyères-le-Châtel, France.
}

\begin{abstract}
High-power accelerators are being studied for several projects including accelerator driven neutron or neutrino sources. The low energy part of these facilities has to be carefully optimized to match the beam requirements of the higher energy parts. The complexity of high intensity beam dynamics in the low energy line is essentially due to the non-linear space charge effects. The PIC code CARTAGO [1] has been developed in order to simulate the beam transport at low energy including the temporal evolution of the space charge compensation. This paper relates new features of the code. The effects of the longitudinal space charge, the image charge and external magnetic field were included. The results of beam transports using solenoid are discussed. Space charge compensation degrees are given.
\end{abstract}

\section{INTRODUCTION}

In the low energy part of an accelerator, a high intensity beam is space charge dominated. Such a beam may be transported in a neutralization regime induced by the charge of the ionized residual gas. This regime occurs naturally when the beam propagates through a residual gas. Gas ionization takes place inside the beam and produces electrons and positive ions. For positive beams, electrons are trapped and ions are repelled to the pipe. The process tends to neutralize the beam space charge.

This paper describes the recent implementations in the code CARTAGO. This PIC code models the coupling between the transverse and the longitudinal beam dynamics including the Space Charge Compensation (SCC) mechanism during the transient and the steady state regimes. An experimental comparison of the code is detailed and discussed. It is based on measurements performed on the Low Energy Beam Transport line (LEBT) of IPHI (Injector of Proton of High Intensity) [2].

\section{NEW FEATURES OF CARTAGO}

The CARTAGO code aims to simulate the effects of the non linear SCC. The algorithm and several of the used numerical methods of the code are detailed in [1]. In order to take into account the effects of the longitudinal space charge induced, for instance, by the divergence of the beam, we modified the Poisson solver. A (r,z) Finite

* Work supported by the European Community-Research Infrastructure Activity under the FP6 "Structuring the European Research Area" programme (CARE, contract number RII3-CT-2003-506395)

${ }^{*}$ bismail $@$,cea.fr
Difference solver has been implemented [3]. The Poisson equation is solved for a charge distribution weighted in a grid with a cloud in cell scheme (CIC). This solver allows to include the image charge.

To perform a better space charge simulation, the secondary beams produced by the source, $\mathrm{H}_{2}{ }^{+}$and $\mathrm{H}_{3}{ }^{+}$, are also included in the calculation. Their contribution is not negligible as they may represent more than $20 \%$ of the total current.

The calculation of the particle trajectories in external dynamic and/or static electromagnetic fields has been implemented. The magnetic field produced by the shielded solenoids of the IPHI-LEBT line is computed with the OPERA-2D code [4].

\section{EXPERIMENTAL SET-UP}

The low energy part of IPHI consists essentially in the light ion source (SILHI), two solenoids for the matching and the transport of the beam and diagnostic section right after the second solenoid [2]. We study, in this paper, the beam dynamics in the first section of the LEBT line. The beam is then stopped by a Faraday cup between the two solenoids. This first section is showed in Fig.1.

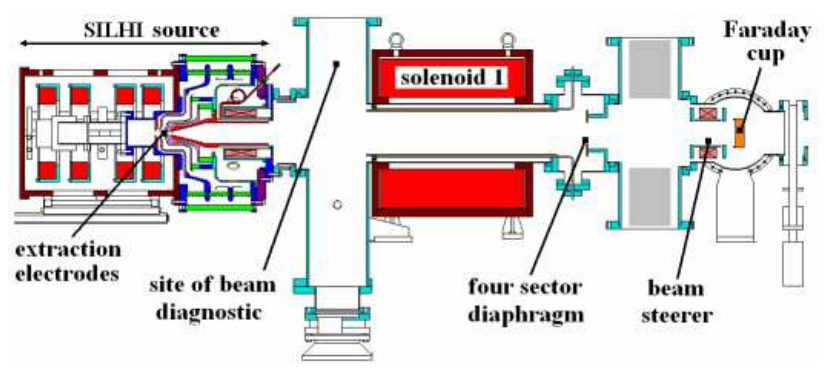

Figure 1: Scheme of the first part of the LEBT line

All measurements were made with a $95 \mathrm{keV}, 100 \mathrm{~mA}$ (total) continuous hydrogen beam produced by the ECR source SILHI. The residual gas pressure near the solenoid 1 was about $5.10^{-5} \mathrm{hPa}$. The measurement of the $\mathrm{H}^{+}, \mathrm{H}_{2}^{+}$and $\mathrm{H}_{3}^{+}$proportions was done with a Wien filter [5]. The measured proportions are respectively $67 \%, 22 \%$ and $7 \%$ for the $\mathrm{H}^{+}, \mathrm{H}_{2}{ }^{+}$and $\mathrm{H}_{3}{ }^{+}$ions. The residual $4 \%$ are composed by heavy ions. These proportions may be improved with an optimisation of the source parameters. 


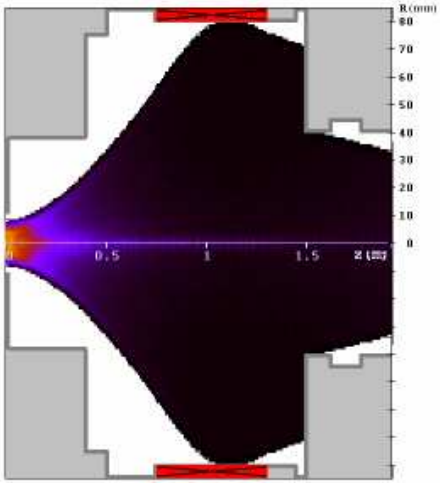

(a) at $0.5 \mu \mathrm{s}$

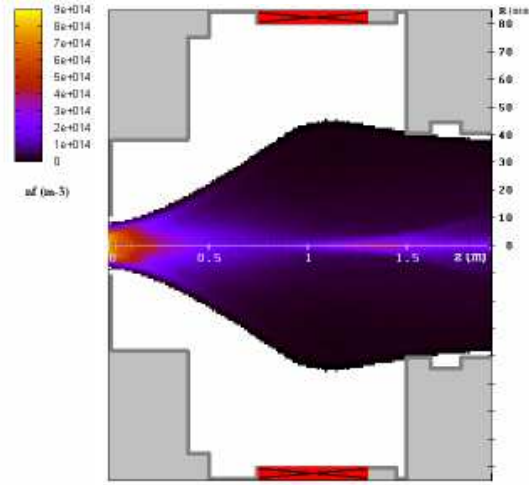

(b) at $2 \mu \mathrm{s}$

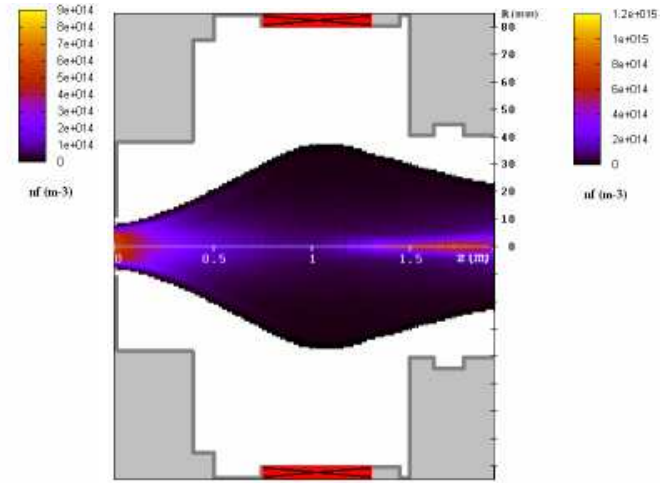

(c) at $4 \mu \mathrm{s}$

Figure 2: The (r,z) proton beam dynamics during the transient regime of the SCC. The current of the solenoid is $150 \mathrm{~A}$.

In order to compare the experimental and the numerical results, we measured several characteristics of the beam at the input and the output of the section. The SCC degree and the beam emittance were measured, between the source and the first solenoid with a four-grid analyser [6] and an emittance measurement unit [5]. The beam transmission as a function of the solenoid focalization is deduced from calorimetric measurements.

\section{COMPARAISON OF THE NUMERICAL AND THE EXPERIMENTAL RESULTS}

To compare the computed steady regime with the measurements, we simulated the dynamics during the entire transient state until the equilibrium is reached. The domain starts at the electron repelling electrode of the source location. We took as initial optical parameters for the beam the output parameters calculated by the code PBGUNS [7]. The beam rms normalized emittance and the Twiss parameters, at the entry of the simulated section, are respectively $\varepsilon_{\mathrm{rms}}=0.04 \pi \mathrm{mm} . \mathrm{mrad}, \alpha=-0.1$ and $\beta=4 \mathrm{~mm} / \pi . \mathrm{mrad}$.

We use the experimental measured proportions to set the relative density of the $\mathrm{H}^{+}, \mathrm{H}_{2}{ }^{+}$and $\mathrm{H}_{3}{ }^{+}$beams at the extraction. The $4 \%$ of heavy ions produced by the source are neglected. We applied a gas pressure of $4.10^{-4} \mathrm{hPa}$ in order to reduce by 10 the time of computation. No significant difference on the SCC mechanism between $4.10^{-4}$ and $4.10^{-5} \mathrm{hPa}$ is expected [1].
The Fig. 2 shows the behaviour of the proton envelop during the transient regime. It appears that the neutralization process is necessary to reach a good transmission with such solenoid (Fig.2a). With a 150 A current in the solenoid 1 , the $H^{+}$beam losses are significantly minimized after $2 \mu$ s of SCC. When reaching the steady regime (at about $6 \mu \mathrm{s}$ ), and due to their relatively important masses, a part of the $\mathrm{H}_{2}{ }^{+}$and $\mathrm{H}_{3}{ }^{+}$ ions are stopped on the four-sector diaphragm (Fig.3.b and 3.c).

During the transient regime, a huge production of secondary particles is recorded in the vacuum chamber. We consider in our simulation, only the production due to the ionisation of the residual gas by the $\mathrm{H}^{+}$and the $\mathrm{H}_{2}^{+}$ beams. The contribution of the $\mathrm{H}_{3}^{+}$is neglected as their proportion and their ionization cross section are weak.

We already studied analytically and numerically the dynamics of the secondary particles, produced by gas ionisation, in a static magnetic and space charge field [8]. We noted that both the electrons and the secondary ions are confined by the magnetic field in the solenoid area. These dynamics are confirmed by the $(r, z)$ simulations of CARTAGO (Fig.4.a and 4.b). Considerable concentrations of electrons and ions are noted inside of the solenoid 1.

More, the traditional mechanism of electron trapping and ion repulsion contribute to the reduction of the electrostatic potential produced by the space charge.

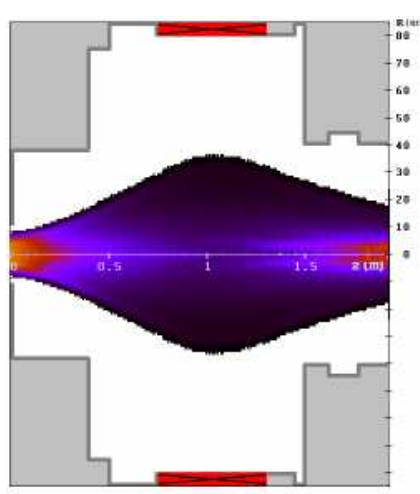

(a) The $H^{+}$beam

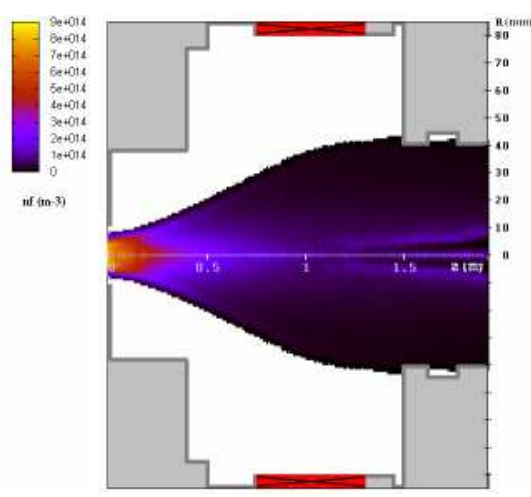

(b) The $\mathrm{H}_{2}^{+}$beam
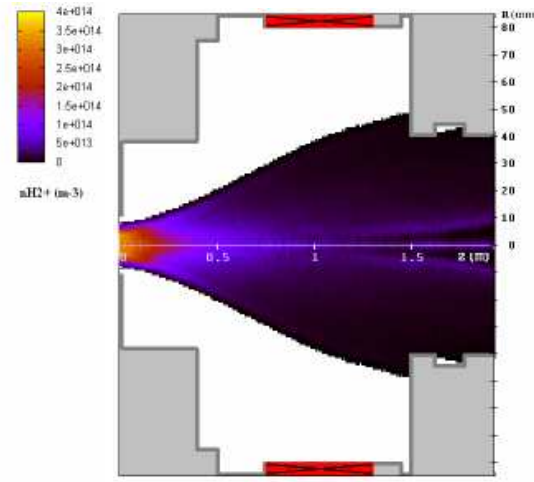

(c) The $\mathrm{H}_{3}{ }^{+}$beam

Figure 3: The (r,z) $\mathrm{H}^{+}, \mathrm{H}_{2}{ }^{+}$and $\mathrm{H}_{3}{ }^{+}$beam dynamics at the steady regime of the SCC (after $6 \mu$ s of computation). 


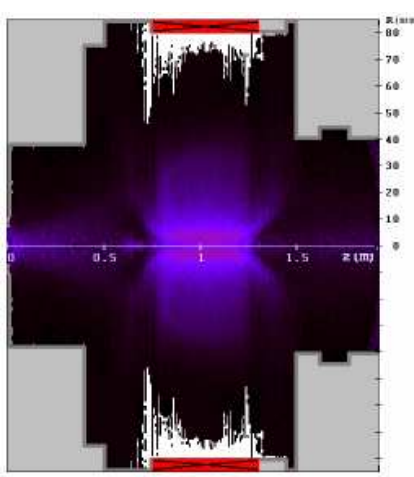

(a) Secondary ion distribution

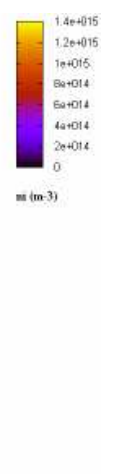

Figure 4: The $(r, z)$ distrib

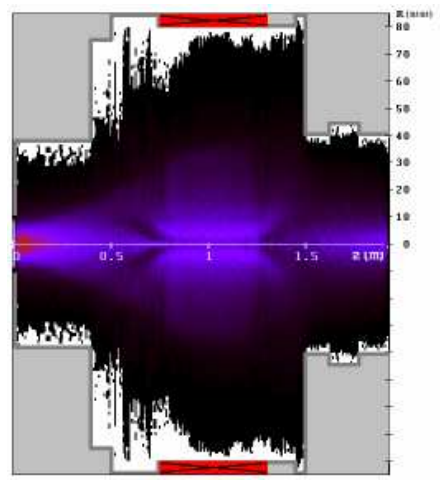

(b) Electron distribution

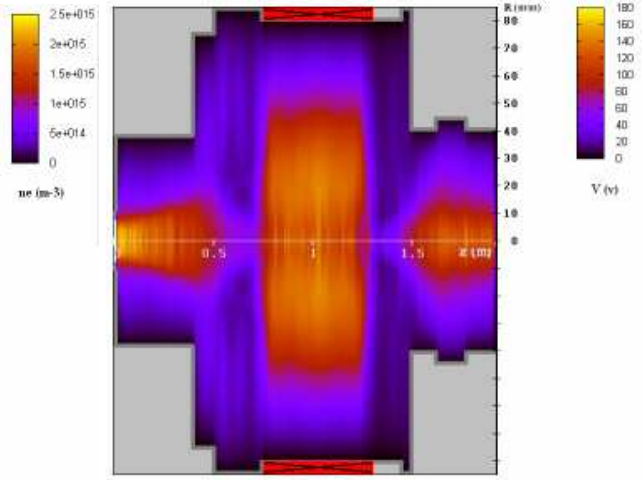

(c) Space charge potential distribution
This potential remains important in some area of the studied section even in the steady state (Fig.4.c). The induced radial and longitudinal space charge forces are non-linear.

To compare these results (the steady regime) with the experimental case, we measured the potential difference between the axis and the edge of the beam $62 \mathrm{~cm}$ after the extraction (between the solenoid 1 and the extraction system). The simulation and the experiment (with the four-grid analyser) give respectively $28.8 \mathrm{~V}$ and $\sim 30 \mathrm{~V}$ when the solenoid 1 is turned off.

Measurements of the beam emittance at this same longitudinal location have been performed with the same experimental conditions. The measurement gave $\varepsilon_{\mathrm{rms}}=0.17 \pi \mathrm{mm} \cdot \mathrm{mrad}(\alpha=-13.2$ and $\beta=9 \mathrm{~mm} / \pi \cdot \mathrm{mrad})$. These values may be compared with the simulated ones: $\varepsilon_{\mathrm{rms}}=0.18 \pi . \mathrm{mm} \cdot \mathrm{mrad}(\alpha=-17.5$ and $\beta=11.5 \mathrm{~mm} / \pi \cdot \mathrm{mrad})$. Several issues could explain the small discrepancy:

- the neglected heavy ions,

- the input beam parameters,

- the approximations used for the measurements (beam with cylindrical symmetry).

A good agreement was recorded (Fig.4) when the numerical and the measured total beam transmission are compared. A set of 7 transmissions has been recorded with the Faraday cup as a function of the solenoid focalization degree.

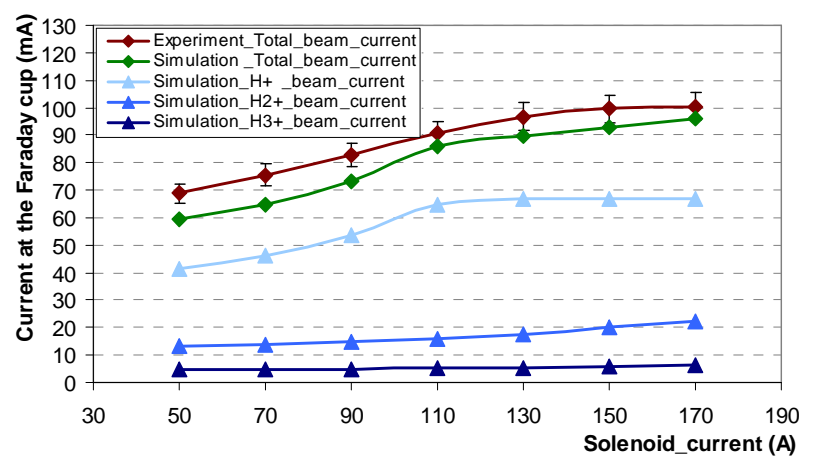

Figure 5: The beam transmission vs. the solenoid current.

\section{CONCLUSION}

2D $(r, z)$ simulations of the first part of the IPHI LEBT line, taking into account SCC effects and secondary beams, have been carried out with the CARTAGO code. The numerical results give a good agreement with the experimental measurements (emittance, total current, beam potential). Future diagnostic developments will be continued in order to refine the comparison with the CARTAGO predictions. In order to obtain a better estimate of the capacity of CARTAGO to predict the beam dynamics in the IPHI LEBT line, more cases will be studied.

\section{ACKNOWLEDGMENTS}

The authors want to thank the SILHI team for their friendly assistance during the LEBT line measurement campaign.

\section{REFERENCES}

[1] A. BenIsmail et al, "Space charge compensation in low energy protons beams", LINAC04, Lubeck.

[2] P.Y. Beauvais, "Status report on the construction of the French High Intensity Proton Injector (IPHI)", EPAC 2002, Paris.

[3] W.H. Press et al, "Numerical Recipes", second edition, Cambridge University Press, 1992.

[4] OPERA-2D, OPERA-3D Vector Fields Ltd, Oxford.

[5] O. Delferriere, "Wien filter for the SILHI source", DSM/GECA/96-16 report, 1996.

[6] R. Ferdinand et al, "Space-charge neutralization measurement of a 75-kev, 130-mA hydrogen-ion beam", PAC97, Canada.

[7] PBGUNS, J.E. Boers, Thunderbird Simulation, Texas.

[8] A. Ben Ismail, "Space charge compensation of high intensity beam", SFP Accelerators Days, october 2003 Porquerolles. 